\title{
Long-Term Effectiveness of Uterosacral Colpopexy and Minimally Invasive Sacral Colpopexy for Treatment of Pelvic Organ Prolapse
}

\author{
Cecile A. Unger, MD, MPH, Matthew D. Barber, MD, MHS, Mark D. Walters, MD, Marie Fidela R. Paraiso, MD, \\ Beri Ridgeway, MD, and J. Eric Jelovsek, MD, MMEd
}

\begin{abstract}
Objectives: The objective of this study was to estimate rates of recurrent pelvic organ prolapse (POP) 6 years after patients underwent transvaginal uterosacral colpopexy, or laparoscopic or robotic sacral colpopexy at a large tertiary care center. We hypothesized that recurrence rates would be higher than those previously reported.

Methods: This is a retrospective study of women who underwent uterosacral colpopexy, laparoscopic, and robotic sacral colpopexy for treatment of POP between 2006 and 2012. A composite outcome for recurrent POP was defined as subjective failure (vaginal bulge symptoms), objective failure (prolapse to or beyond the hymen), or any retreatment for POP (reoperation or use of a pessary). Kaplan-Meier survival curves were generated from each patient's date of follow-up, and parametric survival modeling was used to estimate recurrent POP over 6 years. Annual estimated recurrence rates by type of colpopexy are reported using the composite and individual definitions for recurrent POP.

Results: One thousand three hundred eighty-one subjects met inclusion criteria: 983 (71.1\%) uterosacral, 256 (18.5\%) laparoscopic, and $142(11.2 \%)$ robotic colpopexies. Median (range) months to failure using composite recurrence were as follows: uterosacral, 17.1 (7.6-41); laparoscopic, 10.1 (4.7-25.1); robotic, 9.7 (1.6-17.2). By year 6 in the model, the estimated composite recurrence rates for the uterosacral colpopexy, robotic, and laparoscopic sacral colpopexy groups were $43 \%$, $49 \%$, and $57 \%$, respectively.

Conclusions: Estimated recurrence rates for uterosacral ligament colpopexy, laparoscopic, and robotic sacral colpopexy may be as high as $40 \%$ to $60 \% 6$ years after surgery.
\end{abstract}

Key Words: pelvic organ prolapse, uterosacral colpopexy, sacral colpopexy

(Female Pelvic Med Reconstr Surg 2016;00: 00-00)

U terovaginal prolapse and posthysterectomy vaginal apex prolapse are common pelvic floor disorders that can negatively impact a woman's self-body image and quality of life. ${ }^{1}$ Surgery to treat symptomatic pelvic organ prolapse (POP) is performed using vaginal or abdominal approaches. Uterosacral colpopexy is a common transvaginal procedure for apical prolapse and can be performed at the time of vaginal hysterectomy. ${ }^{2}$ Abdominal sacral colpopexy is considered by many to be the criterion standard for vaginal apex prolapse repair, and in the current literature, has demonstrated superior anatomic outcomes compared with vaginal suspension procedures. ${ }^{3}$ However, abdominal sacral

From the Cleveland Clinic, Obstetrics, Gynecology \& Women's Health Institute, Center for Urogynecology and Pelvic Reconstructive Surgery, Cleveland, $\mathrm{OH}$.

Reprints: Cecile A. Unger, MD MPH, Cleveland Clinic, Obstetrics,

Gynecology \& Women's Health Institute, Center for Urogynecology

and Pelvic Reconstructive Surgery, 9500 Euclid Avenue/A81, Cleveland,

OH 44195. E-mail: ungerc@ccf.org.

The authors have declared they have no conflicts of interest.

Copyright (C) 2016 Wolters Kluwer Health, Inc. All rights reserved.

DOI: $10.1097 / \mathrm{SPV} .0000000000000313$ colpopexy is associated with higher morbidity, increased cost, and longer recovery time when compared with the transvaginal route. Robotic and conventional laparoscopic sacral colpopexy are alternatives to the open abdominal approach and are intended to combine the advantages of transvaginal surgery with comparable success rates of open sacral colpopexy. ${ }^{4-7}$

There is a need for medium to long-term comparative effectiveness studies examining more current vaginal and minimally invasive approaches for prolapse surgery. Sacrospinous colpopexy has been compared with and found to be as anatomically effective as open abdominal sacral colpopexy. ${ }^{8}$ There are 2 randomized controlled studies and several observational studies comparing robotic and laparoscopic sacral colpopexy, ${ }^{9-12}$ and there are several studies that compare minimally invasive abdominal sacral colpopexy (robotic and laparoscopic) to open abdominal sacral colpopexy. ${ }^{6,7,13,14}$ The recently published comparison of 2 transvaginal surgical approaches and perioperative behavioral therapy for apical vaginal prolapse (OPTIMAL trial) found no significant differences in outcomes between patients undergoing transvaginal uterosacral colpopexy and those undergoing sacrospinous colpopexy. ${ }^{15}$ Although efficacy outcomes data for each route exist, few studies followed outcomes beyond 2 years.

The objective of this study was to estimate rates of recurrent POP 6 years after surgery among patients who underwent transvaginal uterosacral colpopexy, laparoscopic, or robotic sacral colpopexy at a large tertiary care referral center. We hypothesized that recurrence rates would be higher than those previously reported, and that rates may differ depending on route of surgery and definition of recurrence.

\section{MATERIALS AND METHODS}

This was a retrospective study of all women who underwent uterosacral colpopexy, or laparoscopic or robotic sacral colpopexy for treatment of POP between 2006 and 2012 at a single tertiary care center. After Institutional Review Board approval was obtained, patients were identified using Current Procedural Terminology codes for intraperitoneal (57283) and sacral (57425) colpopexy. Both sacral colpopexy and uterosacral colpopexy were performed with or without concomitant hysterectomy. Patients were included whether or not they underwent additional concomitant pelvic floor repairs (anterior and/or posterior repair, anal sphincteroplasty, proctopexy, or any anti-incontinence surgery such as midurethral sling placement). Women who underwent uterosacral colpopexy using a laparoscopic approach or extraperitoneal colpopexy (ie, sacrospinous fixation) were not included.

Once patients were identified, the health care system-wide electronic medical record was queried for baseline patient characteristics and intraoperative and postoperative data. Follow-up times were defined as the last visit at which each patient was evaluated by a gynecology provider. Because the definition of recurrent POP that is chosen can result in a high degree of variability 
in recurrence rates, we defined recurrent POP primarily using a composite definition but also reported recurrence using each of the individual criteria used in the composite definition. ${ }^{16}$ The composite outcome for recurrent POP was defined as subjective failure that included a symptomatic feeling of a vaginal bulge, objective failure defined as any prolapse of the vaginal walls to or beyond the hymen during maximum Valsalva, or any retreatment (reoperation or pessary) for POP. These definitions are similar to definitions previously reported in large epidemiologic studies looking at prevalence and trends of $\mathrm{POP}^{17}$ as well as multicenter surgical trials including the recently published Extended Colpopexy and Urinary Reduction Efforts Trial (E-CARE), which looked at the long-term rates of recurrent POP after open abdominal sacral colpopexy. ${ }^{18}$

Four of our 6 surgeons performed minimally invasive abdominal sacral colpopexy during the 2006 to 2012 study period. Each provider used 2 pieces of light-weight polypropylene mesh and either no. 0 or 2-0 polypropylene and/or polydioxanone sutures to attach the mesh to the anterior and posterior vaginal wall. The mesh was sutured to the anterior longitudinal ligament at the level of S1-2 with 2-3 permanent No. 0 or 2-0 polypropylene sutures. ${ }^{19}$ During the study period, all 6 surgeons performed uterosacral colpopexy. Uterosacral colpopexy was performed using 3 techniques that differed based on surgeon suture choice and placement: In the first technique, a 0 polydioxanone and a 0 polypropylene suture were placed through the uterosacral ligament at the level of the ischial spine bilaterally for a total of 4 sutures ( 2 on each side, 1 permanent and 1 delayed absorbable $)^{20}$; in the second modification, three 0 polydioxanone sutures were placed through the uterosacral ligament at the level of ischial spine bilaterally for a total of 6 sutures ( 3 on each side, all delayed-absorbable). ${ }^{3}$ The third technique was a modified high McCalls culdoplasty, and a 0 polydioxanone suture was placed bilaterally through the uterosacral ligament at the level of the ischial spine, followed by placement of one McCall's suture using a 0 polypropylene suture, or $2 \mathrm{McC}$ all's sutures using 0 polydioxanone and 0 polypropylene. Concomitant procedures for all 3 types of colpopexy included hysterectomy (total or supracervical), salpingoophorectomy, anterior and/or posterior repair, anal sphincteroplasty, proctopexy, and/or midurethral sling placement. All surgeries were performed in an academic training center with the assistance of resident and fellow trainees.

Statistical analysis was performed using similar techniques for estimating long-term recurrent POP rates reported in the E-CARE Trial. ${ }^{18}$ Patient characteristics were compared using $\chi^{2}$ and $t$ tests where appropriate. Kaplan-Meier survival curves were generated. Parametric survival models were used to estimate risk of recurrence beyond each patient's actual follow-up time, and yearly estimates of recurrence up to 6 years were reported. Estimated recurrent POP rates for each year of follow-up were performed by classifying women who experienced recurrent

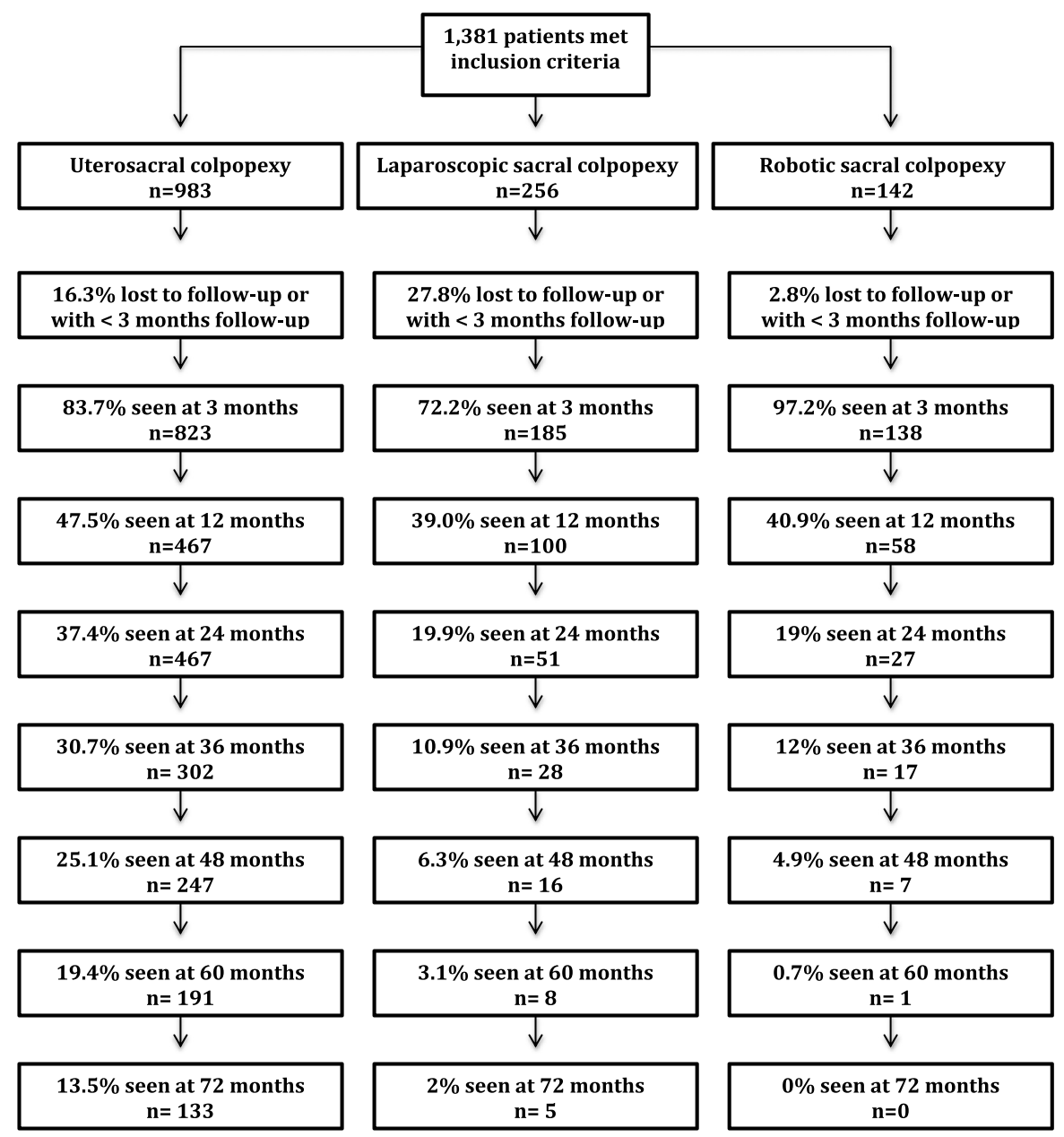

FIGURE 1. Patient Follow-Up by Route of Surgery 
TABLE 1. Patient Characteristics and Concomitant Procedures at the Time of Colpopexy

\begin{tabular}{|c|c|c|c|c|}
\hline & $\mathrm{UC}, \mathbf{n}=983$ & $\mathrm{LSC}, \mathrm{n}=\mathbf{2 5 6}$ & RSC, $n=142$ & $\boldsymbol{P}$ \\
\hline Age $( \pm \mathrm{SD}), \mathrm{y}$ & $61(11)$ & $57(10)^{*}$ & $60(9)$ & $0.001 *$ \\
\hline $\mathrm{BMI}( \pm \mathrm{SD}), \mathrm{kg} / \mathrm{m}^{2}$ & $28.1(5.2)$ & $27.8(5.0)$ & $27.3(4.3)$ & 0.16 \\
\hline Vaginal parity (median [range]) & $3[0-10]$ & $2[0-7]$ & $2[0-6]^{*}$ & 0.16 \\
\hline POP stage (median [range]) & $3[2-4]$ & $3[2-4]$ & $3[2-4]^{*}$ & 0.08 \\
\hline Stage $2, \%$ & 36.0 & 34.5 & 41.5 & \\
\hline Stage $3, \%$ & 60.3 & 58.0 & 55.6 & \\
\hline Stage $4, \%$ & 3.7 & 7.5 & 2.8 & \\
\hline POP-Q C point (median [range]) & $0[-7-+14]^{*}$ & $-1[-8-+8]$ & $-1[-8-+9]$ & $<0.001^{*}$ \\
\hline POP-Q Ba point (median [range]) & $+2[-3-+14]^{*}$ & $+2[-3-+8]$ & $+2[-3-+9]$ & $<0.001^{*}$ \\
\hline POP-Q GH (median [range]) & $4[1-8]^{*}$ & $4[2-8]$ & $4[1-7]$ & $0.01 *$ \\
\hline Previous POP surgery, $\%$ & $6.8^{*}$ & 36.3 & 40.1 & $<0.001^{*}$ \\
\hline Menopause, $\%$ & 82.2 & 77.7 & $88.0^{*}$ & $0.04^{*}$ \\
\hline Tobacco, $\%$ & 7.5 & 7.4 & 8.5 & 0.29 \\
\hline Hysterectomy, \% & $88.2 *$ & 28.9 & 19.7 & $<0.001^{*}$ \\
\hline Anterior repair, \% & $80.0^{*}$ & 6.6 & 8.5 & $<0.001^{*}$ \\
\hline Posterior repair, \% & 58.1 & 49.2 & $33.8^{*}$ & $<0.001^{*}$ \\
\hline Midurethral sling, \% & 59.7 & 66.4 & 62.0 & 0.15 \\
\hline
\end{tabular}

$* P$ value statistically significant at $\leq 0.05$.

UC, uterosacral colpopexy; LSC, laparoscopic sacral colpopexy; RSC, robotic sacral colpopexy; BMI, body mass index; GH, genital hiatus.

POP as failures for each definition used at the last follow-up and for each type of colpopexy. The numerators in all estimates included all treatment failures up to the follow-up time point and denominators included all treatment failures plus known successes at that time point. Women lost to follow-up without documentation of experiencing a treatment failure were censored. Best model fit was determined through graphical information and the Akaike information criterion. ${ }^{21}$ Because our primary aim was not to compare rates of recurrent POP between groups but rather to estimate the rates of time to recurrence for each colpopexy type to guide future comparative trials, we adjusted for certain clinical and statistical prognostic factors that could bias the risk of postoperative recurrence, as well as surgeon preference for 1 approach to surgery versus another. These factors (age, vaginal parity, previous POP surgery, POP Quantification (POP-Q) C point and concomitant posterior repair) were included in the model if they were deemed clinically important in estimating recurrent POP prognosis regardless of whether or not they were independently statistically associated with time to recurrence using $P$ values. All analyses and curves were created using JMP 10.0.2. Two-sided $P$ values of less than 0.05 were considered statistically significant.

\section{RESULTS}

One thousand three hundred eighty-one subjects met inclusion criteria including $983(71.1 \%)$ uterosacral colpopexy cases, $256(18.5 \%)$ laparoscopic sacral colpopexy cases, and 142 $(11.2 \%)$ robotic sacral colpopexy cases. Follow-up ranged from 1 to 28 months with median (interquartile range range) followup time for each group: uterosacral colpopexy, 6.2 (1.5-27.6) months; laparoscopic sacral colpopexy, 5.8 (1.9-13.5) months; robotic sacral colpopexy, $5.8(2.8-13.8)$ months. Figures 1 is a flow diagram of all patients and displays follow-up for each colpopexy type.

Patient characteristics and concomitant procedures for each group are presented in Table 1. Subjects in the laparoscopic group were younger than the robotic (mean difference, 3.7;
$95 \%$ confidence interval $[95 \% \mathrm{CI}], 2.2-5.2)$ and uterosacral (mean difference, 2.9 years; 95\% CI, 1.1-5.1) patients, and subjects in the robotic group were more likely to be menopausal compared with both the laparoscopic (mean difference, $10.3 \%$; 95\% CI, 7.2-12.1) and uterosacral subjects (mean difference, $5.8 \%$; $95 \%$ CI, 3.1-8.9). Subjects in the robotic group had slightly less severe prolapse, because there were more subjects in that group with stage 2 POP compared with the other 2 groups; however, the median POP stage for all 3 groups was 3 (2-4). The uterosacral group had more severe anterior and apical prolapse than the 2 minimally invasive abdominal cohorts, as demonstrated by POP-Q points $\mathrm{C}$ and $\mathrm{Ba}$ and are displayed in Table $1.88 .2 \%$ of the subjects in the uterosacral colpopexy cohort underwent concomitant vaginal hysterectomy, whereas only $28.9 \%$ and $19.7 \%$ of the subjects underwent hysterectomy in the laparoscopic and robotic sacral colpopexy cohorts, respectively,

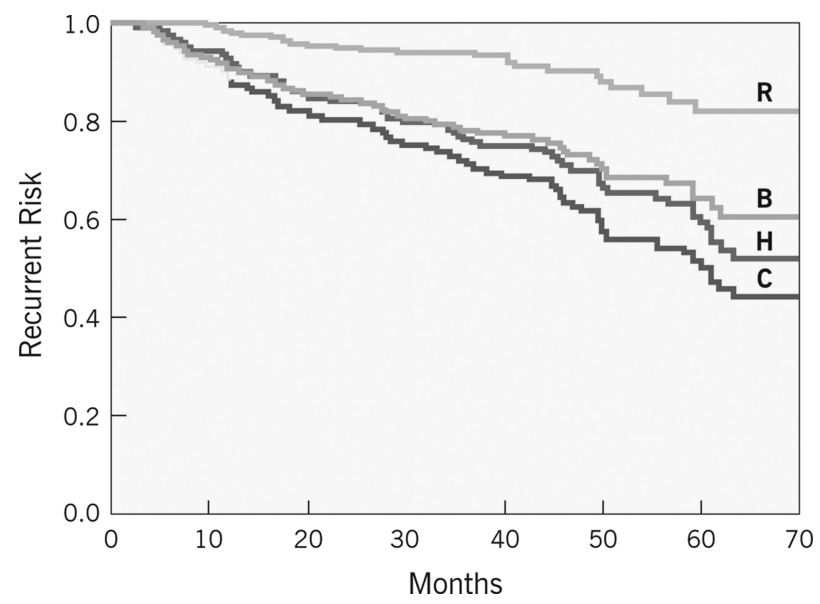

FIGURE 2. Risk of recurrent POP up to 6 years after transvaginal uterosacral colpopexy. $R$, retreatment; $B$, vaginal bulge symptoms; $\mathrm{H}$, prolapse at or beyond the hymen; $\mathrm{C}$, composite definition of recurrent POP. 


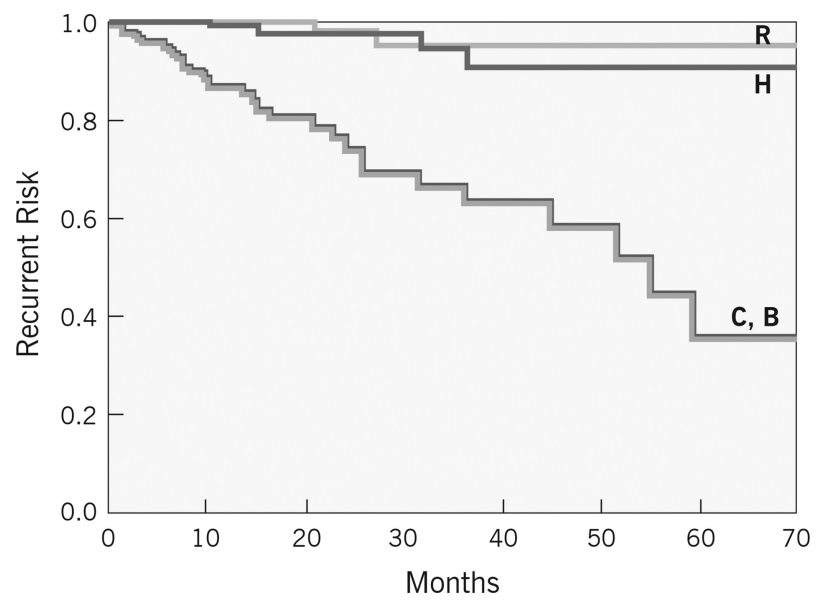

FIGURE 3. Risk of recurrent POP up to 6 years after laparoscopic sacral colpopexy.

because the majority of sacrocolpopexies were performed for posthysterectomy vaginal vault prolapse. Patients undergoing uterosacral colpopexy were more likely to undergo concomitant anterior repair ( $80 \%$ vs $6.6 \%$ vs $8.5 \%, P=<0.001)$, and patients undergoing robotic sacral colpopexy were less likely than both the laparoscopic and uterosacral groups to undergo concomitant posterior repair $(33.8 \%$ vs $49.2 \%$ vs $58.1 \%, P=<0.001)$.

Kaplan-Meier survival curves were generated using all definitions of recurrent POP for each mode of surgery in Figures 2 to 4 . The median (range) estimated time to failure for each definition of POP for uterosacral colpopexy was as follows: composite recurrence, 17.1 (7.6-41) months; vaginal bulge symptoms, 16.3 (6.9-37.1) months; prolapse to or beyond the hymen, 17.6 (8.9-42.4) months; retreatment for POP, 20.1 (12.1-47.2) months. The median (range) estimated time to failure for each definition of POP for laparoscopic sacral colpopexy was: composite recurrence, 10.1 (4.7-25.1) months; vaginal bulge symptoms, 10.1 (4.7-25.1) months; prolapse to or beyond the hymen, 23.6 (11.5-35.3) months; retreatment for POP, 27.4 (21-74.9) months. The median (range) estimated time to failure for each definition of POP for robotic sacral colpopexy was: composite recurrence, 9.7 (1.6-17.2) months; vaginal bulge symptoms, 10 (3.2-21) months; prolapse to or beyond the hymen, 3.5 (1.7-10.7) months; retreatment for POP, 19.1 (6.3-36.7) months.

Table 2 shows the recurrence rates using all definitions for all 3 groups estimated annually over a 6 -year period after surgery. Rates were adjusted for age, vaginal parity, previous POP surgery, POP-Q C point, and concomitant posterior repair. By year 6 , the estimated composite recurrence rates for the uterosacral colpopexy, robotic and laparoscopic sacral colpopexy groups were $43 \%, 49 \%$, and $57 \%$, respectively. Rates of symptomatic bulge were $34 \%, 44 \%$, and $49 \%$. Rates of POP to or beyond the hymen were $34 \%, 11 \%$, and $22 \%$ and rates of retreatment for POP were $14 \%, 8 \%$, and $23 \%$ for uterosacral colpopexy, laparoscopic, and robotic sacral colpopexy, respectively.

A subanalysis examining the different uterosacral techniques as well as the surgeons performing each technique did not show a difference in the above outcomes (data not shown). In the robotic cohort, $86 \%$ of retreatment for POP was for symptomatic rectocele, and 14\% were for symptomatic cystocele. In the laparoscopic group, $67 \%$ were for symptomatic rectocele, and $33 \%$ were for symptomatic cystocele. There were no retreatments for apical prolapse in both the robotic and laparoscopic groups. In the uterosacral colpopexy cohort, $34 \%$ were for rectocele, $55 \%$ were for cystocele, and $15 \%$ were for apical prolapse with or without rectocele and/or cystocele.

\section{DISCUSSION}

Previously published observational studies have looked at postoperative POP recurrence ${ }^{22}$; however, this cohort study is the largest to date examining 3 commonly performed approaches to the repair of apical POP (MEDLINE search from 1998 to March 2015). In this study, each experienced surgeon performed the approach deemed to be the best and most appropriate by both patient and surgeon. Using a strict composite definition, estimated recurrence rates for all 3 modes may be as high as $40 \%$ to $60 \%$ 6 years after surgery. Although these data were obtained from a retrospective cohort of patients, it is important to recognize the value of evidence that can come from nonrandomized surgical trials evaluating surgical interventions when the conduct of randomized surgical trials may be impractical. ${ }^{23}$ Historically, randomized surgical trials comparing different approaches to treating apical POP have been difficult to conduct due to surgeon proficiency in 1 technique and patient and or surgeon approach preference. Since 2013, only 6 randomized trials $(N=568)$ with patients receiving surgery for apical POP reported a subjective failure outcome using prolapse symptoms; and, there have been 8 trials $(\mathrm{N}=663)$ reporting objective failure after apical prolapse surgery. ${ }^{3}$ No trials have compared traditional vaginal approaches to minimally invasive approaches for the repair of apical POP and follow-up for all trials has generally been less than 2 years. This study is a valuable addition to our knowledge of surgical outcomes after POP surgery because it provides 6-year estimates of subjective, objective, and retreatment recurrence using over 1000 POP surgeries performed by experienced surgeons.

Our high recurrence estimates should not be surprising. In 2013, the E-CARE Trial revealed similarly high estimated rates of recurrent POP over time in patients who underwent open abdominal sacral colpopexy, ${ }^{18}$ and revealed that the rates of recurrence were much higher than previously reported with the open approach. Seven years after open abdominal sacral colpopexy, estimated rates of symptomatic failure were between $29 \%$ and $24 \%$, anatomic failure rates were between $27 \%$ and $22 \%$, and composite rates of failure were $48 \%$ and $34 \%$ for their 2 groups. In 2014, Barber et al published the findings of the randomized controlled comparison of 2 transvaginal surgical approaches and perioperative behavioral therapy for apical

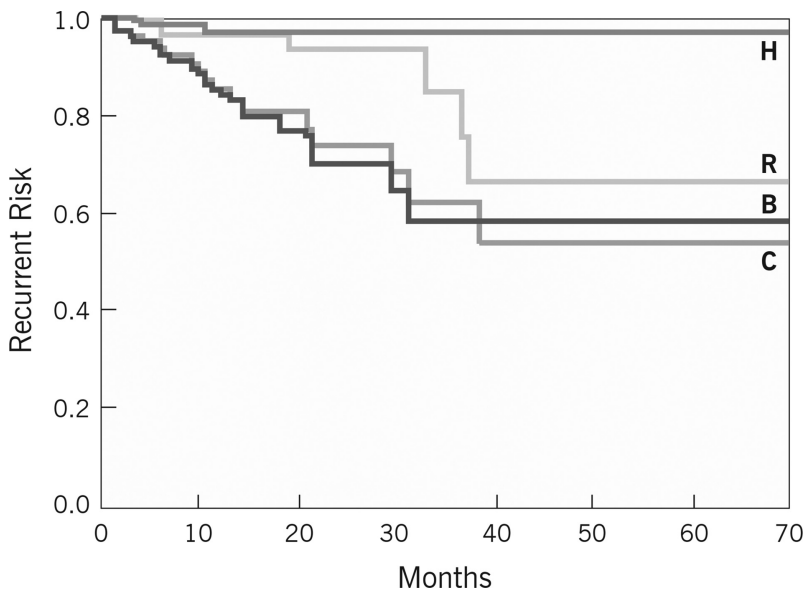

FIGURE 4. Risk of recurrent POP up to 6 years after robotic sacral colpopexy. 
TABLE 2. Estimated Probability of Recurrent POP After Transvaginal Colpopexy and Minimally Invasive Sacral Colpopexy Using Composite and Individual Definitions*

\begin{tabular}{|c|c|c|c|c|}
\hline Recurrent POP Definition & & UC \% $(95 \%$ CI $)$ & LSC \% $(95 \%$ CI $)$ & RSC \% $(95 \%$ CI $)$ \\
\hline Bulge & $1 \mathrm{y}$ & $6.0(4.2-8.4)$ & $9.9(5.9-15.7)$ & $12.4(7.0-20.2)$ \\
\hline \multirow[t]{5}{*}{ Symptoms } & $2 \mathrm{y}$ & $13.3(9.9-17.2)$ & $19.9(13.1-28.6)$ & $23.7(14.9-34.7)$ \\
\hline & $3 \mathrm{y}$ & $19.6(15.2-24.6)$ & $27.8(19.1-38.1)$ & $32.3(21.5-44.8)$ \\
\hline & $4 y$ & $24.9(19.7-30.9)$ & $34.4(24.3-45.4)$ & $39.1(27.0-52.3)$ \\
\hline & $5 y$ & $29.7(23.7-36.2)$ & $39.6(28.8-51.2)$ & $44.6(31.7-58.1)$ \\
\hline & $6 y$ & $33.8(27.3-40.8)$ & $44.1(32.6-56.0)$ & $49.2(35.8-62.7)$ \\
\hline Prolapse to & $1 \mathrm{y}$ & $4.6(3.0-6.8)$ & $0.7(0.1-2.3)$ & $2.0(0.5-6.2)$ \\
\hline Or beyond & $2 y$ & $11.6(8.3-15.6)$ & $2.3(0.6-6.7)$ & $5.9(1.9-14.7)$ \\
\hline \multirow[t]{4}{*}{ Hymen } & $3 \mathrm{y}$ & $18.2(13.6-23.5)$ & $4.3(1.3-11.2)$ & $10.1(3.6-22.4)$ \\
\hline & $4 y$ & $24.0(18.5-30.4)$ & $6.6(2.2-15.7)$ & $14.2(5.5-29.2)$ \\
\hline & $5 \mathrm{y}$ & $29.2(22.8-36.4)$ & $8.8(3.2-19.9)$ & $18.0(7.5-34.9)$ \\
\hline & $6 y$ & $33.8(26.7-41.6)$ & $11.1(4.2-23.8)$ & $21.6(9.4-40.0)$ \\
\hline \multirow[t]{6}{*}{ Retreatment } & $1 \mathrm{y}$ & $1.0(0.4-2.3)$ & $0.3(0.1-2.0)$ & $3.2(0.8-8.5)$ \\
\hline & $2 y$ & $3.8(2.0-6.4)$ & $1.3(0.1-5.9)$ & $8.3(3.0-17.1)$ \\
\hline & $3 y$ & $6.7(3.9-10.4)$ & $2.8(0.4-9.6)$ & $12.7(5.3-23.5)$ \\
\hline & $4 y$ & $9.4(5.8-14.0)$ & $4.5(0.8-13.0)$ & $16.5(7.5-28.5)$ \\
\hline & $5 \mathrm{y}$ & $11.9(7.5-17.3)$ & $6.1(1.4-16.0)$ & $19.8(9.5-32.6)$ \\
\hline & $6 y$ & $14.1(9.1-20.3)$ & $7.6(1.9-18.7)$ & $22.6(11.4-36.1)$ \\
\hline \multirow[t]{6}{*}{ Composite } & $1 \mathrm{y}$ & $7.9(5.8-10.7)$ & $10.3(6.2-16.1)$ & $14.2(8.3-22.3)$ \\
\hline & $2 y$ & $17.7(13.9-22.1)$ & $21.6(14.5-30.5)$ & $27.6(18.2-38.9)$ \\
\hline & $3 y$ & $25.9(20.9-31.3)$ & $30.7(21.6-41.1)$ & $37.6(26.3-50.2)$ \\
\hline & $4 y$ & $32.7(26.9-38.8)$ & $37.9(27.7-49.1)$ & $45.4(32.9-58.2)$ \\
\hline & $5 \mathrm{y}$ & $38.4(32.1-45.0)$ & $43.9(32.9-55.5)$ & $51.5(38.5-64.4)$ \\
\hline & $6 y$ & $43.3(36.5-50.2)$ & $48.9(37.4-60.6)$ & $56.5(43.2-69.1)$ \\
\hline
\end{tabular}

*Rates adjusted using survival models to control for age, vaginal parity, previous POP surgery, POPQ C point, and concomitant posterior repair.

vaginal prolapse and reported a surgical success rate (as defined by a composite outcome measure) over 2 years of $64.5 \%$ for transvaginal uterosacral colpopexy. In this study, we used similar statistical models used in the E-CARE study to estimate the long-term rates of recurrent $\mathrm{POP}$ after uterosacral colpopexy, laparoscopic, and robotic sacral colpopexy, and we found similar recurrence rate estimates 6 years after surgery. Together, these results highlight a potential need to shift our paradigm regarding what we know about the natural history of prolapse recurrence, and this may change how we communicate about expectations after POP surgery. These results may be used to help counsel patients about the long-term efficacy of these surgeries.

We recognize that the 3 groups evaluated in this study differed in their baseline characteristics. The majority of patients receiving uterosacral colpopexy were primary prolapse repairs, whereas up to $40 \%$ of the laparoscopic and robotic cases had undergone previous POP surgery. Despite our attempts to control for baseline differences including previous POP surgery, we did find differences among the groups, but we urge clinicians to use caution in making comparisons between approaches using these data.

For instance, when baseline risk factors (such as previous POP surgery and concomitant rectocele repair) for recurrent POP were controlled for, uterosacral colpopexy appeared to have a higher risk of anatomic recurrent POP but a lower risk of symptomatic bulge and need for retreatment for POP when compared with robotic and laparoscopic sacral colpopexy. When robotic and laparoscopic sacral colpopexy were compared, the robotic cases had a much higher risk of need for retreatment for recurrent POP, but similar subjective symptoms and slightly higher anatomic recurrence (most commonly in the posterior compartment). In 2005, our group showed that laparoscopic sacral colpopexy was more likely to be associated with posterior compartment recurrence compared with open abdominal sacral colpopexy. ${ }^{7}$ In a recently published meta-analysis of outcomes after robotic sacral colpopexy, Hudson et $\mathrm{al}^{24}$ found that the most common reason for reoperation after robotic sacral colpopexy was recurrent prolapse with a combined estimated rate of $3.3 \%(95 \% \mathrm{CI}, 1.4-5.2)$ with the majority being posterior repairs for nonapical prolapse. Our data show that recurrence is also more common in the posterior compartment after minimally invasive abdominal cases compared with transvaginal cases. It is possible that sacral colpopexy may be more effective in the apex and proximal vagina, whereas transvaginal repairs may be more effective in the distal vagina. Furthermore, recurrence in the proximal vagina after transvaginal surgery may not be noticeable until it reaches the hymen, and as a result, smaller defects may not be very bothersome to patients, which may be why there are fewer complaints of vaginal bulge in this group. Conversely, smaller defects in the distal vagina may be more common after sacral colpopexy and may be more bothersome to patients. This is likely to be a result of anatomic location or a wider genital hiatus, but may also be associated with other pelvic floor symptoms such as incontinence, voiding dysfunction, outlet constipation, and chronic straining during defecation.

It is unclear why patients in this study report more symptomatic bulge after sacral colpopexy, but have less anatomic failure compared with transvaginal colpopexy. The axis of the vagina 
after suspension is different between the 2 procedures, and it is possible that suspension to the sacrum after sacral colpopexy may lead to different postoperative sensations in the vagina that are interpreted as bulge and pressure by patients. It is also possible that sacral colpopexy itself is a risk factor for distal symptomatic failures. We believe that the compartment that is opposite to the mesh-reinforced compartment is likely to be the "at risk" compartment for recurrence, and this may hold true for the distalproximal axis as well. If the proximal vagina is mesh-reinforced, but the distal aspect is not, patients may perceive the movement associated with the non-mesh-reinforced area of the vagina as sensations of bulge and pressure, when in fact, there is no anatomic recurrence. This study and others demonstrate that there are differences in anatomic and subjective recurrence patterns between routes of surgery, and we may need to start explaining to patients that outcomes are route-dependent. Future research in this area is necessary.

Strengths of this study are the size of the cohort and the use of different definitions of recurrence that include objective and subjective outcomes. In addition, the use of parametric survival modeling allowed us to calculate estimated recurrence rates after controlling for baseline risk factors and to account for different follow-up times as well as the differential slopes of failure over time for each definition of recurrence. These are an improvement over point estimate rates and could be used to guide future surgical trials in this area. A limitation of time to event analysis is that we must presume that once a patient meets 1 definition of recurrence, the patient is always considered a recurrence (ie, the outcome under study remains static and does not change over time). Patient reported outcomes related to POP are a dynamic process and may worsen or improve over time in patients. Therefore, the estimated recurrence rates in this study may overestimate true recurrence rates. The most significant limitation of this study is its retrospective design. Follow-up was not prospectively determined which may bias toward those subjects who returned for evaluation of recurrent POP or with other complaints. We cannot account for subjects who presented to outside facilities for recurrence, and we do not have POP-Q examinations on patients beyond their last visit with their provider. In addition, treatment estimates among the 3 approaches could be explained by our inability to control for unmeasured risk factors leading to measurement bias. There may be inherent differences between the 3 groups that could have led the surgeons to designate patients to 1 group and not the other. Although we did control for baseline risk factors that were likely to contribute to our outcomes, (age, vaginal parity, previous POP surgery, POPQ C point, and concomitant posterior repair), other differences could have altered our results but could not be controlled for due to our retrospective study design. In addition, we were unable to control for surgeon as a confounder as not all surgeons performed all 3 operations. Despite these limitations, our findings are similar to those found in the E-CARE study for patients who underwent open abdominal sacral colpopexy, and add to the data that currently exist on POP recurrence after apical prolapse repair.

Estimated recurrence rates for uterosacral ligament colpopexy, laparoscopic, and robotic sacral colpopexy may be as high as $40 \%$ to $60 \% 6$ years after surgery, with $34 \%$ to $49 \%$ developing symptomatic recurrence and $8 \%$ to $23 \%$ undergoing retreatment. This is higher than previously reported in studies with shorter follow-up but consistent with long-term estimates for open abdominal sacral colpopexy. Comparisons of recurrence after POP surgery should be substantially longer than 1 or 2 years because there appear to be differential rates of recurrence between approaches and should include subjective and objective definitions of recurrence. Future studies should investigate which outcomes are most important to patients to guide valid comparisons between approaches for POP surgery.

\section{REFERENCES}

1. Jelovsek JE, Barber MD. Women seeking treatment for advanced pelvic organ prolapse have decreased body image and quality of life. Am J Obstet Gynecol 2006;194:1455-1461.

2. Shull BL, Bachofen C, Coates KW, et al. A transvaginal approach to repair of apical and other associated sites of pelvic organ prolapse with uterosacral ligaments. Am J Obstet Gynecol 2000;183(6):1365-1373.

3. Maher C, Feiner B, Baessler K, et al. Surgical management of pelvic organ prolapse in women. Cochrane Database of Syst Rev 2013. Issue 4. Art. No.: CD004014. DOI: 10.1002/14651858.CD004014.pub5.

4. Ganatra AM, Rozet F, Sanchez-Salas R, et al. The current status of laparoscopic sacrocolpopexy: a review. Eur Urol 2009;55(5): 1089-1103.

5. Benson JT, Lucente V, McClellan E. Vaginal versus abdominal reconstructive surgery for the treatment of pelvic support defects: a prospective randomized study with long-term outcome evaluation. Am J Obstet Gynecol 1996;175(6):1418-1421.

6. Geller EJ, Parnell BA, Dunivan GC. Robotic vs abdominal sacrocolpopexy: a 44-month pelvic floor outcomes. Urology 2012; 79(3):532-536.

7. Paraiso MF, Walters MD, Rackley RR, et al. Laparoscopic and abdominal sacral colpopexies: a comparative cohort study. Am J Obstet Gynecol 2005;192(5):1752-1758.

8. Maher CF, Qayawneh AM, Dwyer PL, et al. Abdominal sacral colpopexy or vaginal sacrospinous colpopexy for vaginal vault prolapse: a prospective randomized study. Am J Obstet Gynecol 2004;190(1): 20-26.

9. Freeman RM, Pantazis K, Thomson A, et al. A randomised controlled trial of abdominal versus laparoscopic sacrocolpopexy for the treatment of post-hysterectomy vaginal vault prolapse: LAS study. Int Urogyncol J 2013;24(3):377-384.

10. Paraiso MF, Jelovsek JE, Frick A, et al. Laparoscopic compared with robotic sacrocolpopexy for vaginal prolapse: a randomized controlled trial. Obstet Gynecol 2011;118(5):1005-1013.

11. Seror J, Yates DR, Seringe E, et al. Prospective comparison of short-term functional outcomes obtained after pure laparoscopic and robot-assisted laparoscopic sacrocolpopexy. World J Urol 2012; 30(3):393-398.

12. Tan-Kim J, Menefee SA, Luber KM, et al. Robotic-assisted and laparoscopic sacrocolpopexy: comparing operative times, costs and outcomes. Female Pelvic Med Reconstr Surg 2011;17(1): 44-49.

13. Siddiqui NY, Geller EJ, Visco AG. Symptomatic and anatomic 1-year outcomes after robotic and abdominal sacrocolpopexy. Am J Obstet Gynecol 2012;206(5):435.e1-5.

14. Khan A, Alperin M, Wu N, et al. Comparative outcomes of open versus laparoscopic sacrocolpopexy among Medicare beneficiaries. Int Urogynecol J 2013;24(11):1883-1891.

15. Barber MD, Brubaker L, Burgio KL, et al. Comparison of 2 transvaginal surgical approaches and perioperative behavioral therapy for apical vaginal prolapse: the OPTIMAL randomized trial. JAMA 2012;311(10): 1023-1034.

16. Barber MD, Brubaker L, Nygaard I, et al. Defining success after surgery for pelvic organ prolapse. Obstet Gynecol 2009;114(3):600-609.

17. Wu JM, Vaughan CP, Good PS, et al. Prevalence and trends of symptomatic pelvic floor disorders in US women. Obstet Gynecol 2014;123(1): 141-148. 
18. Nygaard I, Brubaker L, Zyczynski HM, et al. Long-term outcomes following abdominal sacrocolopexy for pelvic organ prolapse. JAMA 2013;309(19):2016-2024.

19. Unger CA, Paraiso MF, Jelovsek JE, et al. Perioperative adverse events after minimally invasive abdominal sacrocolpopexy. Am J Obstet Gynecol 2014;211(5):547.e1-547.e8.

20. Barber MD, Visco AG, Weidner AC, et al. Bilateral uterosacral ligament vaginal vault suspension with site-specific endopelvic fascia defect repair for treatment of pelvic organ prolapse. Am J Obstet Gynecol 2000;183:1402-1410.
21. Akaike H. A new look at the statistical model identification. IEEE Transact Automatic Control 1974;19(6):716-723.

22. Whiteside JL, Weber AM, Meyn LA, et al. Risk factors for prolapse recurrence after vaginal repair. Am J Obstet Gynecol 2004;191(5): 1533-1538.

23. Farrokhyar F, Karanicolas PJ, Thoma A, et al. Randomized controlled trials of surgical interventions. Ann Surg 2010;251(3):409-416.

24. Hudson CO, Northington GM, Lyles RH, et al. Outcomes of robotic sacrocolpopexy: a systematic review and meta-analysis. Female Pelvic Med Reconstr Surg 2014;20(5):252-260. 